\title{
BMJ Open Hospital variation in allogeneic transfusion and extended length of stay in primary elective hip and knee arthroplasty: a cross-sectional study
}

\author{
Veronique M A Voorn, ${ }^{1}$ Perla J Marang-van de Mheen, ${ }^{1}$ Anja van der Hout, ${ }^{1}$ \\ Cynthia So-Osman, ${ }^{2,3}$ M Elske van den Akker-van Marle, ${ }^{1}$ \\ Ankie W M M Koopman-van Gemert, ${ }^{4}$ Albert Dahan, ${ }^{5}$ Thea P M Vliet Vlieland, ${ }^{6}$ \\ Rob G H H Nelissen, ${ }^{6}$ Leti van Bodegom-Vos, ${ }^{1}$ on behalf of the LISBOA study \\ group
}

To cite: Voorn VMA, Marangvan de Mheen PJ, van der Hout A, et al. Hospital variation in allogeneic transfusion and extended length of stay in primary elective hip and knee arthroplasty: a crosssectional study. BMJ Open 2017;7:e014143. doi:10.1136/ bmjopen-2016-014143

- Prepublication history and additional material are available. To view these files please visit the journal online (http://dx.doi. org/10.1136/bmjopen-2016014143).

Received 2 September 2016 Revised 12 April 2017 Accepted 18 April 2017

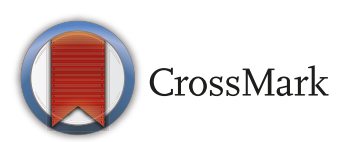

For numbered affiliations see end of article.

Correspondence to Dr Leti van Bodegom-Vos; I. vanbodegom-vos@lumc.nl

\section{ABSTRACT}

Objectives Outcomes in total hip and knee arthroplasty (THA and TKA), such as allogeneic transfusions or extended length of stay (LoS), can be used to compare the performance of hospitals. However, there is much variation in these outcomes. This study aims to rank hospitals and to assess hospital differences of two outcomes in THA and TKA: allogeneic transfusions and extended LoS, and to additionally identify factors associated with these differences.

Design Cross-sectional medical record review study. Setting Data were gathered in 23 Dutch hospitals. Participants 1163 THA and 986 TKA patient admissions. Outcomes Hospitals were ranked based on their observed/expected (O/E) ratios regarding allogeneic transfusion and extended LoS percentages (extended LoS was defined by postoperative stay $>4$ days). To assess the reliability of these rankings, we calculated which percentage of the existing variation was based on differences between hospitals as compared with random variation (after adjustment for variation in patient characteristics). Associations between hospital-specific factors and 0/E ratios were used to explore potential sources of differences.

Results The variation in 0/E ratios between hospitals ranged from 0 to 4.4 for allogeneic transfusion, and from 0.08 to 2.7 for extended LoS. Variation in transfusion could in $21 \%$ be explained by hospital differences in THA and 34\% in TKA. For extended LoS this was 71\% in THA and 78\% in TKA. Better performance (low 0/E ratios) in transfusion was associated with more frequent tranexamic acid (TXA) use in TKA ( $R=-0.43, p=0.04)$. Better performance in extended LoS was associated with more frequent TXA use in THA ( $R=-0.45, p=0.03)$ and TKA $(R=-0.65, p<0.001)$ and local infiltration analgesia $(L I A)$ in TKA ( $R=-0.60, p=0.002)$.

Conclusions Ranking hospitals based on allogeneic transfusion is unreliable due to small percentages of variation explained by hospital differences. Ranking based on extended LoS is more reliable. Hospitals using TXA and LIA have relatively fewer patients with transfusions and extended LOS.
Strengths and limitations of this study

- This study is the first that uses the rankability concept to assess hospital differences for a blood management indicator among orthopaedic patients.

- Ranking on outcome indicators should be interpreted carefully as the ability to assess real hospital differences is limited in case of a low rankability.

- A possible limitation of this study is that we were not able to take factors that are difficult to express in a number into account as potential sources for hospital differences, for instance: the use of discharge and rehabilitation protocols of each hospital, the use of risk assessment tools.

\section{INTRODUCTION}

In conditions such as osteoarthritis and rheumatoid arthritis, total hip arthroplasty (THA) and total knee arthroplasty (TKA) are widely accepted treatments and recommended in many guidelines. ${ }^{12}$ In 2011, up to 160 per 100000 persons received a THA and 119 per 100000 persons received a TKA in high-income countries. ${ }^{3}$ From several studies it is known that there is a large variation in outcomes for THA and TKA between hospitals, such as allogeneic transfusion rates, length of stay $(\mathrm{LoS})$, readmission rates or revision rates. ${ }^{4-8}$ This variation consists of three components: variation caused by the mix of patient characteristics present within a hospital, variation caused by differences between hospitals and random variation.

In order to gain insight into the differences between hospitals, rankings are used to compare hospitals on different outcome indicators. $^{9-12}$ However, such rankings are only reliable if they are based on true hospital differences and with little random 
variation. $^{9}{ }^{12-15}$ Therefore, the reliability of ranking hospitals on specific outcomes should be verified. Reliable rankings can be used as a starting point to look for explanatory factors and consequently to improve the performance of hospitals. ${ }^{116}$

In this study hospitals were ranked on two frequently used short-term outcome indicators and it was assessed whether ranking on these outcomes is reliable. The outcome indicators were allogeneic red blood cell transfusion percentage and extended LoS in patients undergoing primary elective THA or TKA.

Although allogeneic transfusions are relatively safe, transfusion reactions, transmission of diseases and immunomodulatory effects resulting in increased susceptibility of infections may occur. ${ }^{17}$ In the Dutch transfusion guideline, the following three thresholds were recommended for adult patients undergoing elective surgery: consider transfusion if the haemoglobin (Hb) level is $<4 \mathrm{mmol} / \mathrm{L}$ $(6.4 \mathrm{~g} / \mathrm{dL})$ in healthy adults (American Society of Anesthesiologists (ASA) physical status classification 1); consider transfusion if the $\mathrm{Hb}$ level is $<5 \mathrm{mmol} / \mathrm{L}(8 \mathrm{~g} / \mathrm{dL})$ in ASA 1 patients $>60$ years and in uncomplicated ASA 2 and ASA 3 patients; consider transfusion if the $\mathrm{Hb}$ level is $<6 \mathrm{mmol} / \mathrm{L}(9.6 \mathrm{~g} / \mathrm{dL})$ in ASA 4 patients, patients who are not able to increase their cardiac output to compensate for haemodilution, patients with sepsis, patients with severe pulmonary disease or patients with symptomatic cerebrovascular disease.$^{18}$ Ninety per cent of Dutch hospitals reported to use this guideline. The remaining $10 \%$ reported to use the guideline with additional blood saving techniques or did not have any transfusions. ${ }^{19}$ Consequently, it seems unlikely that variability in allogeneic transfusions between hospitals is explained by variability in following the guidelines. Therefore, the allogeneic transfusion rate may be considered as a robust outcome indicator to judge hospital performance. A low percentage of allogeneic transfusion is therefore pursued by both physicians, insurance companies and regulators. ${ }^{20}$

Another indicator for good quality of care is the absence of complications. Given that extended LoS may be caused by the occurrence of complications, it is thus often used as an indicator for quality of care. ${ }^{91}$ Both outcome indicators are used frequently as short-term outcomes for studies regarding all kinds of interventions in the fields of patient blood management and joint replacement research, ${ }^{22-24}$ and are used as quality indicators to assess hospital performance or for cost calculations. ${ }^{20}$ 25-27

To improve a hospitals' ranking, it is important to know which factors account for the differences between hospitals after adjustment for the mix of patients within a hospital. In the literature, a number of patient characteristics were found to be associated with the outcomes allogeneic transfusion and LoS such as age, gender, body mass index (BMI), smoking status, diagnosis, preoperative $\mathrm{Hb}$ or ASA classification. ${ }^{28}{ }^{29}$ In addition to these patient characteristics a number of hospital-specific factors, such as the use of blood management techniques, ${ }^{30-35}$ enhanced recovery programmes, ${ }^{36}{ }^{37}$ the type of anaesthesia, ${ }^{38-40}$ the use of cement in THA, ${ }^{41}$ the surgical approach in $\mathrm{THA}^{42}$ and the use of a tourniquet in TKA, ${ }^{43} 44$ were identified from literature. Association between these factors and better performance of hospitals give starting points to improve quality of care.

The aim of this study is to rank hospitals based on 'allogeneic transfusions' and 'extended LoS', to assess which part of the variation in the outcomes 'allogeneic transfusions' and 'extended LoS' is due to true differences between hospitals and to identify hospital-specific factors associated with these between-hospital differences in patients undergoing THA or TKA.

\section{MATERIALS AND METHODS}

\section{Study design and setting}

In this retrospective study, the medical records of patients undergoing a primary elective THA or TKA were reviewed. The used data were gathered for the baseline measurement of the 'Leiden Implementation Study of Blood management in hip and knee arthroplasty' (LISBOA) trial, ${ }^{45}$ a cluster randomised trial in which a deimplementation strategy on blood management among THA and TKA patients was implemented and evaluated. The LISBOA trial is registered with the Dutch trial register www.trialregister.nl (ID: NTR4044). Data were gathered in 23 non-academic Dutch hospitals. A sample of approximately 100 patients in each hospital, undergoing surgery from May through October 2013, was selected (the first 20 THA/TKA procedures of a month in a 5-month period, as this was the time frame of the baseline measurement of the LISBOA trial). Fifteen hospitals were located in the western urban part of the Netherlands, the other eight hospitals were located in more rural areas. Included were 9 teaching hospitals, 13 general hospitals and 1 private clinic. A mean number of 6.2 orthopaedic surgeons were employed per hospital ranging from 3 to 10 orthopaedic surgeons. All participating hospitals reported to follow the Dutch transfusion guideline ${ }^{18}$

\section{Population}

Patients included in the LISBOA baseline measurement underwent primary elective THA or TKA and were $\geq 18$ years. Exclusion criteria consisted of usual exclusion criteria for elective orthopaedic surgery including patients with a serious disorder of the coronary, peripheral and/or carotid arteries, recent myocardial infarction or cerebrovascular accident in the past 6 months, patients with untreated hypertension (diastolic $>95 \mathrm{~mm} \mathrm{Hg}$ ), patients with a pregnancy, patients with anaemia $(\mathrm{Hb}<10 \mathrm{~g} / \mathrm{dL})$ and exclusion criteria specifically used for this study, including bilateral surgery (within 6 weeks), patients with a malignancy (except skin cancer and cured cancer), patients with a coagulation disorder, patients refusing or with a contraindication for allogeneic blood transfusions.

\section{Data collection}

For each patient admission, the following characteristics were collected: age, gender, preoperative $\mathrm{Hb}$ (in g/ 
$\mathrm{dL}$ ), preoperative diagnosis (osteoarthritis, rheumatoid arthritis or other), BMI (in $\mathrm{kg} / \mathrm{m}^{2}$ ), smoking status (yes or no) and ASA classification (1, 2, 3, 4). For each patient admission data were also collected on treatment factors determined by the hospital (hospital-specific factors): type of anaesthesia (general vs locoregional), use of cement (yes or no), surgical approach in THA (anterior vs other), use of a tourniquet in TKA (yes or no), use of preoperative erythropoietin (EPO) (yes or no), intra or postoperative use of cell salvage system (yes or no), use of tranexamic acid (TXA) (yes or no), use of local infiltration analgesia (LIA) (yes or no). These factors can be seen as patient specific, but the choice to use them is mainly included in hospital-specific treatment protocols or selected for most patients within a hospital and were therefore included as hospital-specific factors.

Finally, the outcomes for each patient admission were assessed: allogeneic transfusion (yes or no) and postoperative LoS (in days, excluding the day of surgery). The outcome indicator 'extended LoS' was defined by the highest quartile of the LoS among all patients. In both THA and TKA patients this was $>4$ postoperative days.

\section{Statistical analyses}

The associations of patient characteristics with the allogeneic transfusion and extended LoS were tested separately for THA and TKA. This was done using multivariate logistic regression models. The included patient characteristic variables were derived from the literature and included: age, gender, BMI, smoking status, diagnosis, preoperative $\mathrm{Hb}$ or ASA classification. ${ }^{28} 29$

Hospitals were ranked by using the observed/ expected $(\mathrm{O} / \mathrm{E})$ ratio for each hospital and for THA and TKA patients separately. For this calculation, the observed outcome was the number of THA or TKA patient admissions with a transfusion or an extended LoS within a hospital. The expected outcome was the sum of all patients' expected probabilities for either a transfusion or extended LoS, adjusted for patient characteristics calculated using a multivariate logistic regression model, fitted on the data of all included hospitals and including all patient characteristic variables.

The observed outcome was divided by the expected outcome resulting in an $\mathrm{O} / \mathrm{E}$ ratio per hospital. For each hospital, the $95 \%$ CIs of the $\mathrm{O} / \mathrm{E}$ ratio were calculated with the Mid-P exact test for Poisson variates. ${ }^{46}$ For an average performing hospital, the observed outcome will equal the expected outcome, resulting in an $\mathrm{O} / \mathrm{E}$ ratio of 1. Hospitals with an $\mathrm{O} / \mathrm{E}$ ratio significantly $<1$ (including its $95 \%$ CI) have significantly fewer events and are therefore performing better than average (positive outliers). Similarly, hospitals with an $\mathrm{O} / \mathrm{E}$ ratio significantly $>1$ are performing worse than average (negative outliers).

Second, we assessed which part of the variation in the outcomes 'allogeneic transfusion' and 'extended LoS' was due to true hospital differences and which part was due to random variation (after adjustment for patient characteristics). This was done by calculating the rankability as an indicator of the reliability of ranking. ${ }^{15}$ This rankability is defined as the between-hospital variation divided by the sum of the between-hospital variation and the within-hospital variation. The between-hospital variation was estimated using the heterogeneity from the random effects logistic regression model in which hospitals were included as random factor and all patient characteristic variables mentioned above as fixed factors. The within-hospital variation was estimated using a fixed effects logistic regression model, including dummy variables for hospital and all patient characteristic variables as fixed factors. The median squared $\mathrm{SE}$ of the coefficient for the hospital variable was used to estimate the within-hospital variation. ${ }^{15}$

The rankability for a particular outcome indicator is expressed as a percentage. High rankability means that a large percentage of the variation is explained by true hospital differences. Rankability increases if the effect of being treated in a hospital can be estimated more precisely (less within-hospital variation) and if the differences between hospitals are larger (more between-hospital variation). Rankability in general will be lower for outcomes with lower event rates as this is usually estimated with lower precision. So within-hospital variation is likely to be larger thereby making it harder to detect between-hospital differences and resulting in lower rankability.

Finally, to explore which hospital factors may be associated with better performance of a hospital (lower $\mathrm{O} / \mathrm{E}$ ratio) on the two outcome indicators, and thereby explain part of the true hospital differences, we calculated Pearson's pairwise correlation coefficients $(\mathrm{R})$ between a hospital factor (percentage of patients exposed to the factor) and the hospital $\mathrm{O} / \mathrm{E}$ ratio. Before calculating the correlation coefficients the data were visually checked for non-linearity by using scatter plots. The following factors were explored: type of anaesthesia, use of cement in THA, surgical approach in THA, use of a tourniquet in TKA, use of preoperative EPO, use of cell salvage, use of TXA, use of LIA.

$p$ Values $<0.05$ were considered significant in all analyses.

The Medical Ethical Committee of the Leiden University Medical Center decided that ethical approval was not required under Dutch National law (CME 13/132). The gathering of patient data is conducted in compliance with the Good Clinical Practices protocol.

\section{RESULTS}

In total, the records of 1163 admissions of patients undergoing THA and 986 admissions of patients undergoing TKA were reviewed in 23 hospitals. Table 1 shows the distribution of patient characteristics and outcomes between hospitals. The number of total patient admissions per hospital ranged from 64 to 100 with a median of 97 (with a median of 51 THA and 42 TKA per hospital). The percentage of patient admissions requiring transfusion varied between hospitals from $1.9 \%$ to $26.1 \%$ in THA and from $0.0 \%$ to $29.2 \%$ in TKA (table 1). The percentage of patient admissions with extended hospital 
Table 1 Distribution of patient characteristics and outcomes in participating hospitals

\begin{tabular}{|c|c|c|c|c|}
\hline & \multicolumn{2}{|c|}{ THA ( $n=23$ hospitals) } & \multicolumn{2}{|c|}{ TKA ( $n=23$ hospitals) } \\
\hline & Median & Range & Median & Range \\
\hline Mean age (years) & 69.2 & $64.9-74.6$ & 69.5 & $66.5-73.7$ \\
\hline Mean BMI $\left(\mathrm{kg} / \mathrm{m}^{2}\right)$ & 27.4 & $25.9-28.7$ & 30.0 & $28.6-31.3$ \\
\hline Smoking & $12.9 \%$ & $8.1 \%-21.3 \%$ & $8.8 \%$ & $6.3 \%-8.0 \%$ \\
\hline ASA 2 & $64.8 \%$ & $51.6 \%-86.0 \%$ & $65.3 \%$ & $47.9 \%-87.8 \%$ \\
\hline ASA 3 & $13.0 \%$ & $0.0 \%-29.0 \%$ & $16.7 \%$ & $2.4 \%-29.7 \%$ \\
\hline ASA $4^{*}$ & $0 \%$ & - & $0 \%$ & $0.0 \%-2.9 \%$ \\
\hline Mean preoperative $\mathrm{Hb}(\mathrm{g} / \mathrm{dL})$ & 13.8 & $13.4-14.2$ & 13.9 & $13.4-14.2$ \\
\hline Allogeneic blood transfusion & $7.0 \%$ & $1.9 \%-26.1 \%$ & $4.1 \%$ & $0.0 \%-29.2 \%$ \\
\hline Mean LoS (days) & 4.2 & $2.1-5.4$ & 4.0 & $2.3-6.5$ \\
\hline Extended LoS (>4 days) & $24.5 \%$ & $1.9 \%-44.4 \%$ & $22.0 \%$ & $2.0 \%-62.5 \%$ \\
\hline
\end{tabular}

The value (either mean or percentage) of the median hospital and range between hospitals are shown.

${ }^{*}$ In all further analyses, ASA 3 and ASA 4 are combined due to the small number of ASA 4 patients $(n=2)$.

ASA, American Society of Anesthesiologists; BMI, body mass index; Hb, haemoglobin; LoS, length of stay; OA, osteoarthritis; RA, rheumatoid arthritis; THA, total hip arthroplasty; TKA, total knee arthroplasty.

stay (>4 postoperative days) ranged from $1.9 \%$ to $44.4 \%$ in THA and from $2.0 \%$ to $62.5 \%$ in patients with TKA between hospitals (table 1 ).

The impact of the different patient characteristic variables on the outcome indicators is shown separately for THA and TKA in online supplementary appendix 1 . Each patient characteristic was significantly associated with at least one of the outcome indicators in either THA or TKA except for smoking.

\section{Variation between hospitals}

All included hospitals were ranked based on their $\mathrm{O} / \mathrm{E}$ ratios (figures 1 and 2 for allogeneic transfusion and figures 3 and 4 for extended LoS). Each hospital is represented by the same letter across figures. The $\mathrm{O} / \mathrm{E}$ ratios between hospitals ranged from 0 to 4.4 for transfusions (figure 1 for THA and figure 2 for TKA) and from 0.08 to

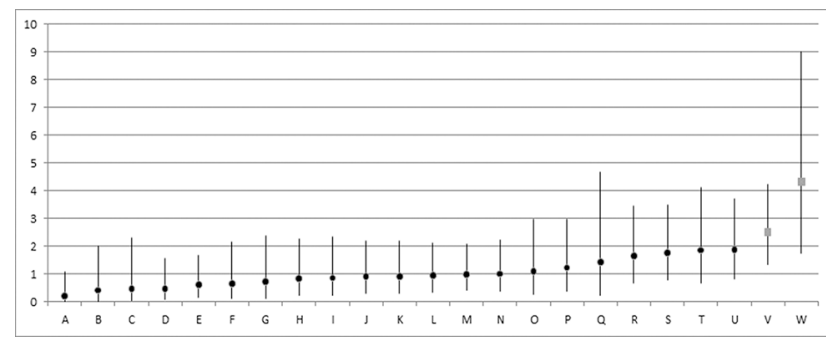

Figure 1 Observed/Expected ranking of hospitals based on allogeneic transfusion in total hip arthroplasty, adjusted for patient characteristics. Each hospital is marked by a letter corresponding to the same hospital across figures. Square dots indicate negative outlier hospitals.
2.7 for extended LoS (figure 3 for THA and figure 4 for TKA). Three hospitals were identified as negative outliers with significantly more transfusions than expected in both THA and TKA (hospitals V and $\mathrm{W}$ in THA, figure 1 , and hospitals V, K and T in TKA, figure 2). For extended LoS after THA, three hospitals were positive outliers (hospitals $\mathrm{A}, \mathrm{C}$ and $\mathrm{O}$ ) and three hospitals were negative outliers (hospitals V, B and G) (figure 3). For extended LoS after TKA, five hospitals were positive outliers (hospitals N, $\mathrm{A}, \mathrm{C}, \mathrm{J}$ and $\mathrm{R}$ ) and five hospitals were negative outliers (hospitals V, E, P, K and T) (figure 4).

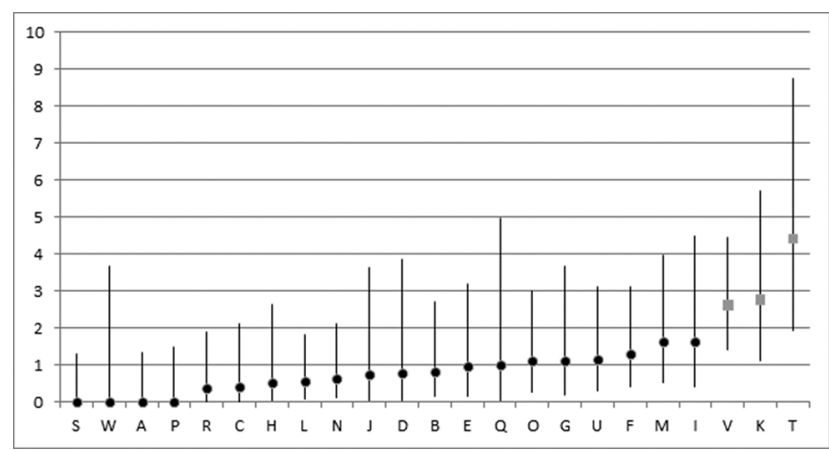

Figure 2 Observed/Expected ranking of hospitals based on allogeneic transfusion in total knee arthroplasty, adjusted for patient characteristics. Each hospital is marked by a letter corresponding to the same hospital across figures. Square dots indicate negative outlier hospitals. 


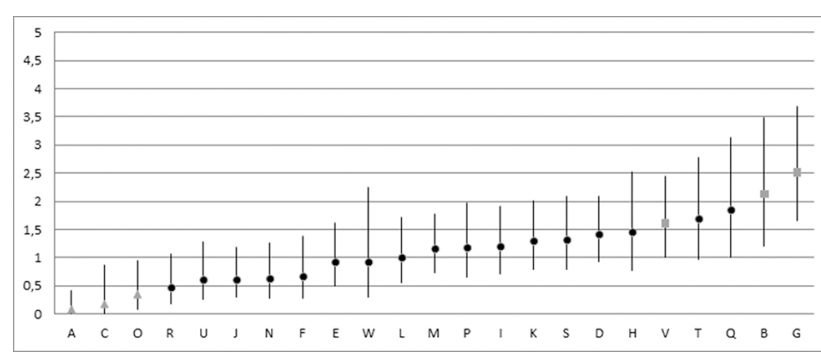

Figure 3 Observed/Expected ranking of hospitals based on extended length of stay in total hip arthroplasty ( $>4$ days) adjusted for patient characteristics. Each hospital is marked by a letter corresponding to the same hospital across figures. Triangle dots indicate positive outlier hospitals, square dots indicate negative outlier hospitals.

\section{Reliability of ranking}

The rankability, to assess the reliability of ranking, is shown in online supplementary appendix 2. The rankability allogeneic transfusion after THA was $21 \%$, meaning that $21 \%$ of the observed differences in transfusions are true hospital differences and $79 \%$ is random variation (due to chance). Rankability of allogeneic transfusion after TKA is $34 \%$. Rankability of percentage of patients with extended LoS is higher, $71 \%$ for THA and $78 \%$ for TKA so that the majority of the observed variation are true differences.

\section{Possible explanatory factors for hospital performance}

To explore which hospital factors could be associated with outcomes, we calculated the correlation coefficients (R) between hospital-specific factors and $\mathrm{O} / \mathrm{E}$ ratios (table 2). In THA patients, more frequent use of TXA within a hospital was associated with a lower $\mathrm{O} / \mathrm{E}$ ratio (which might indicate better performance) for extended LoS $(\mathrm{R}=-0.45, \mathrm{p}=0.03)$. In TKA, more frequent TXA use was significantly associated with both a lower allogeneic transfusion percentage than expected $(\mathrm{R}=-0.43$, $\mathrm{p}=0.04)$ and fewer patients with extended $\operatorname{LoS}(\mathrm{R}=-0.65$, $\mathrm{p}<0.001)$. In addition, more frequent use of LIA in TKA patients was associated with fewer patients with extended $\operatorname{LoS}(\mathrm{R}=-0.60, \mathrm{p}=0.002)$. No other factors were identified to be associated with hospital performance.

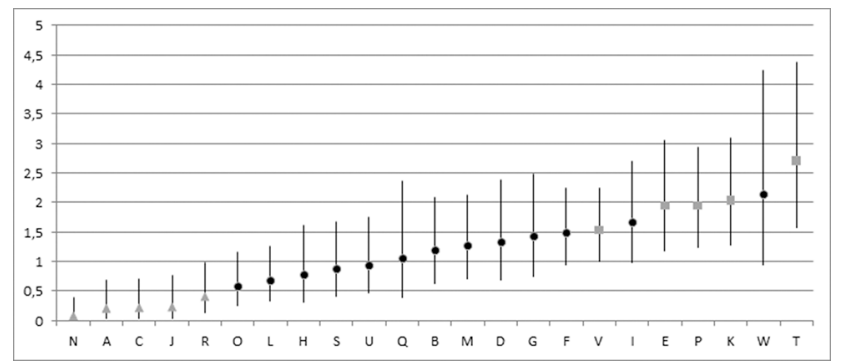

Figure 4 Observed/Expected ranking of hospitals based on extended length of stay in total knee arthroplasty ( $>4$ days) adjusted for patient characteristics. Each hospital is marked by a letter corresponding to the same hospital across figures. Triangle dots indicate positive outlier hospitals, square dots indicate negative outlier hospitals.

\section{DISCUSSION}

This study has shown that the reliability of ranking hospitals on differences in their allogeneic transfusion percentage is low, especially for THA in which $21 \%$ of the variation between hospitals reflects true differences, but also for TKA $(34 \%)$. The reliability of ranking hospitals based on differences in extended LoS is better $(71 \%$ in THA and 78\% in TKA) compared with allogeneic transfusion percentages. Thus, this indicator is more suitable for ranking hospitals. Hospitals using TXA more frequently have fewer patients with extended LoS in both THA and TKA and fewer allogeneic transfusions in TKA patients. Furthermore, hospitals in which LIA is used more frequently also have fewer patients with extended LoS in TKA.

Ranking seems to be a simple and pragmatic way to get insight into variation between hospitals. However, ranking on outcome indicators should be interpreted carefully as the ability to assess real hospital differences is limited in case of a low rankability. This study is the first that uses the rankability concept, as introduced by Van Houwelingen et $a l^{47}$ for a blood management indicator among orthopaedic patients. A cut-off point for the rankability to indicate whether an indicator is reliable does not exist. Lingsma et $a l^{48}$ suggest that rankability $>70 \%$ is fair to rank hospitals. van Dishoeck et al ${ }^{15}$ use the same categorisation as the $\mathrm{I}^{2}$ of heterogeneity in meta-analyses, which is similar in nature to the rankability measure. This categorisation assigned low, moderate and high to the $\mathrm{I}^{2}$ values $25 \%, 50 \%$ and $75 \% .{ }^{49}$ With the latter categorisation the rankability of our outcome indicators is low for allogeneic transfusion and moderate to high for extended LoS. Following these suggestions, ranking hospitals based on allogeneic transfusion percentages should not be pursued as it is too unreliable, and this outcome indicator should therefore be preserved for individual monitoring and quality improvement purposes. Ranking based on percentage extended LoS is more reliable. However, any categorisation of rankability is still considered arbitrary as already pointed out by others. ${ }^{15} 48$

Furthermore, caution is needed regarding the interpretation of $\mathrm{O} / \mathrm{E}$ ranking and rankability. The $\mathrm{O} / \mathrm{E}$ statistic will in theory show how much the number of events in a hospital differs from an idealised or fictive value based on the patient characteristics of that hospital. As such it expresses the magnitude of differences between hospitals and can be used to rank the hospitals. The rankability quantifies the reliability of ranking. However, the exact statistical properties and sensitivity for underlying between-hospital differences of both measures should be investigated in further research.

A possible limitation may be lack of power given the number of patients included per hospital in this study. However, given the observed average transfusion rate of $7 \%$ for THA and median number of 51 patients per hospital, identifying a $14 \%$ transfusion rate with $80 \%$ power and $95 \%$ reliability would require 18 hospitals (assuming an 
Table 2 Correlation coefficients between hospital factor and outcomes in THA and TKA adjusted for patient characteristics

\begin{tabular}{lllll}
\hline Hospital factor & $\begin{array}{l}\mathbf{R} \text { (p value) } \\
\text { allogeneic } \\
\text { transfusion in THA }\end{array}$ & $\begin{array}{l}\mathbf{R} \text { (p value) } \\
\text { extended LoS in }\end{array}$ & $\begin{array}{l}\mathbf{R} \text { (p value) } \\
\text { Tllogeneic } \\
\text { transfusion in TKA }\end{array}$ & $\begin{array}{l}\text { R (p value) } \\
\text { extended LoS in } \\
\text { TKA }\end{array}$ \\
\hline Preoperative EPO (if $\mathrm{Hb}<13.0 \mathrm{~g} / \mathrm{dL})$ & $-0.27(0.21)$ & $-0.10(0.64)$ & $-0.10(0.66)$ & $-0.10(0.66)$ \\
General anaesthesia (vs regional) & $-0.24(0.24)$ & $-0.01(0.97)$ & $0.22(0.30)$ & $-0.01(0.95)$ \\
Use of LIA & $-0.12(0.59)$ & $-0.27(0.21)$ & $-0.30(0.15)$ & $-0.60(0.002)$ \\
Use of tranexamic acid & $-0.14(0.53)$ & $-0.45(0.03)$ & $-0.43(0.04)$ & $-0.65(<0.001)$ \\
Use of a tourniquet & - & - & $-0.21(0.34)$ & $-0.20(0.36)$ \\
Minimal invasive anterior approach & $-0.04(0.86)$ & $-0.00(1.0)$ & - & - \\
(vs lateral/posterolateral approach) & & & - & - \\
Cemented acetabulum & $-0.32(0.14)$ & $-0.22(0.31)$ & - & - \\
Cemented femur & $-0.34(0.11)$ & $-0.23(0.28)$ & - & $0.18(0.42)$ \\
\hline Cell salvage system & $-0.24(0.28)$ & $-0.18(0.42)$ & $-0.11(0.61)$ & - \\
\hline
\end{tabular}

EPO, erythropoietin; Hb, haemoglobin; LIA, local infiltration analgesia; LoS, length of stay; THA, total hip arthroplasty; TKA, total knee arthroplasty.

intraclass correlation of 0.01$)$. Such a difference is small in the total range that we report (from $1.9 \%$ to $26.1 \%$, see table 1) and we have data from more hospitals, so that is likely that the number of THA patients has been sufficient. Similarly for TKA with a median number of 42 patients per hospital, we would be able to identify a difference between $4 \%$ and $10 \%$ with 20 hospitals. For LoS, the range between hospitals was even larger. Therefore, we think it is not likely that a lack of power has been a major problem in identifying between-hospital differences.

The observed variation between hospitals may be determined by a different general perioperative policy between hospitals, different surgical techniques and treatment protocols and, in the case of allogeneic transfusion, by different blood management strategies. We tried to identify specific hospital factors that are associated with better or worse performance on allogeneic transfusion and extended LoS. A higher percentage of patients treated with TXA or LIA is associated with better performing hospitals on extended LoS and allogeneic transfusion. The use of TXA and LIA is frequently incorporated in broader programmes such as 'enhanced recovery' or 'fast track, ${ }^{39} 50$ which are developed to optimise care and reduce LoS, so this may be part of the explanation of the associations found.

A second limitation of this study is that we were not able to measure in which individual patients 'enhanced recovery' or 'fast track' protocols were used. However, we did measure the use of LIA and TXA, which are frequently part of these protocols. In addition, other factors that possibly contribute to the differences between hospitals, that are difficult to express in a number, were not taken into account in this study. For instance: the discharge and rehabilitation protocols of each hospital, regional differences, differences in the amount of complex cases, the use of risk assessment tools, the effect of resident participation or the use of dedicated operating rooms ${ }^{51-55}$ were not included.
In future research, other outcome indicators that are both relevant for clinical practice and reliable for rankings should be identified. Frequently used outcomes such as infection rate, 30-day readmission rate and revision rate should be tested whether these are reliable enough to make valid between-hospital rankings. Optionally, outcome indicators could be combined to increase the rankability (eg, extended LoS with readmission). In general, outcomes with low event rates will be estimated with larger imprecision and thus larger within and between-hospital variation, mostly resulting in low rankability. Therefore, it seems better to pursue outcome indicators with sufficient numbers of events. In addition, the identification and implementation of hospital-specific factors associated with better performance should be further expanded to improve performance of hospitals and to increase quality of care for the patients.

\section{Author affiliations}

${ }^{1}$ Department of Medical Decision Making, Leiden University Medical Center, Leiden, The Netherlands

${ }^{2}$ Department of Transfusion Medicine, Sanquin Blood Supply, Leiden, The Netherlands

${ }^{3}$ Department of Internal Medicine, Groene Hart Hospital, Gouda, The Netherlands ${ }^{4}$ Department of Anesthesiology, Albert Schweitzer Hospital, Dordrecht, The Netherlands

${ }^{5}$ Department of Anesthesiology, Leiden University Medical Center, Leiden, The Netherlands

${ }^{6}$ Department of Orthopedics, Leiden University Medical Center, Leiden, The Netherlands

Acknowledgements We would like to acknowledge all orthopaedic departments of the hospitals that participated in the LISBOA project (alphabetically): Alrijne Hospital, Leiden; Alrijne Hospital, Leiderdorp; Bravis Hospital, Bergen op Zoom; Bravis Hospital, Roosendaal; Canisius Wilhelmina Hospital, Nijmegen; Diaconessenhuis, Meppel; Gelderse Vallei Hospital, Ede; IJsselland Hospital, Capelle aan den IJssel; Isala Clinics, Zwolle; Maartenskliniek, Woerden; Meander Medical Centre, Amersfoort; Medisch Spectrum Twente, Enschede; Onze Lieve Vrouwe Gasthuis, Amsterdam; Orthopaedic Clinic Orthopedium, Delft; Rijnstate Hospital, Arnhem; Rivas Beatrix Hospital, Gorinchem; Sint Franciscus Hospital, Rotterdam; St Lucas Andreas Hospital, Amsterdam; Tergooi Hospitals, Hilversum; VieCuri Medical Centre, Venlo; Zuyderland MC, Sittard-Geleen. 
Contributor LISBOA study group: B Boetes, SJM Breugem, GA Haeseker, D Haverkamp, JA Jansen, YE Könst, NP Kort, CALC Kremers-van de Hei, G Meermans, JJ Nieuwenhuis, AA Polak, WJ Rijnberg, WWJ Rosenberg, VAB Scholtes, H Sonneveld, M Stegeman, A Swets, EJ Uitterlinden, SBW Vehmeijer, AMJS Vervest, J Wolkenfelt, AVCM Zeegers, RG Zuurmond.

Contributors The study was designed by VV, PM, CS, TV, RN and LB. Data gathering was executed by $\mathrm{V}$ and $\mathrm{AH}$. Interpretation and analysis of data was done by $\mathrm{V}, \mathrm{PM}, \mathrm{AH}, \mathrm{CS}, \mathrm{MA}, \mathrm{AK}, \mathrm{RN}, \mathrm{PM}$ and LB. WV drafted the manuscript. All authors critically revised the manuscript and approved the final version.

Funding This work was supported by the Netherlands Organisation for Health Research and Development grant number 171203001 and Sanquin Blood Supply grant number PPOC13-010. Funding was obtained from the Netherlands Organisation for Scientific Research (NWO) Incentive fund Open Access publications. The funding sources did not play a role in the investigation.

Competing interests None declared.

Patient consent The Medical Ethical Committee of the Leiden University Medical Center decided that ethical approval was not required under Dutch National law (CME 13/132). The gathering of patient data is conducted in compliance with the Good Clinical Practices protocol.

Ethics approval The Medical Ethical Committee of the Leiden University Medical Center.

Provenance and peer review Not commissioned; externally peer reviewed.

Data sharing statement The relevant anonymised data are available upon reasonable request from the authors.

Open Access This is an Open Access article distributed in accordance with the Creative Commons Attribution Non Commercial (CC BY-NC 4.0) license, which permits others to distribute, remix, adapt, build upon this work non-commercially, and license their derivative works on different terms, provided the original work is properly cited and the use is non-commercial. See: http://creativecommons.org/ licenses/by-nc/4.0/

(C) Article author(s) (or their employer(s) unless otherwise stated in the text of the article) 2017. All rights reserved. No commercial use is permitted unless otherwise expressly granted.

\section{REFERENCES}

1. Dutch Institute for Healthcare Improvement CBO. Guideline for diagnostis and treatment in hip- and knee arthritis (in Dutch). CBO: Utrecht, 2007.

2. Nelson AE, Allen KD, Golightly YM, et al. A systematic review of recommendations and guidelines for the management of osteoarthritis: the chronic osteoarthritis management initiative of the U.S. bone and joint initiative. Semin Arthritis Rheum 2014:43:701-12.

3. OECD. Health at a Glance 2013: OECD indicators. 2015. www.oecdilibrary.org.

4. Siciliani L, Sivey P, Street A. Differences in length of stay for hip replacement between public hospitals, specialised treatment centres and private providers: selection or efficiency? Health Economics 2013;22:234-42.

5. Menendez ME, Lu N, Huybrechts KF, et al. Variation in use of blood transfusion in primary total hip and knee arthroplasties. $J$ Arthroplasty 2016;31:2757-63.

6. Bozic KJ, Grosso LM, Lin Z, et al. Variation in hospital-level riskstandardized complication rates following elective primary total hip and knee arthroplasty. J Bone Joint Surg Am 2014;96:640-7.

7. Solomon DH, Losina E, Baron JA, et al. Contribution of hospital characteristics to the volume-outcome relationship: dislocation and infection following total hip replacement surgery. Arthritis and Rheumatology 2002;46:2436-44.

8. So-Osman C, Nelissen R, Te Slaa R, et al. A randomized comparison of transfusion triggers in elective orthopaedic surgery using leucocyte-depleted red blood cells. Vox Sang 2010;98:56-64.

9. Cram P, Cai X, Lu X, et al. Total knee arthroplasty outcomes in topranked and non-top-ranked orthopedic hospitals: an analysis of Medicare administrative data. Mayo Clin Proc 2012;87:341-8.

10. Olmsted M G, Geisen E, Murphy J, et al. Methodology: U.S. News \& World Report best hospitals 2014-15. North Carolina: RTI International, 2015.

11. Robertsson O, Ranstam J, Lidgren L. Variation in outcome and ranking of hospitals: an analysis from the Swedish knee arthroplasty register. Acta Orthop 2006;77:487-93.
12. van Dishoeck AM, Koek MB, Steyerberg EW, et al. Use of surgicalsite infection rates to rank hospital performance across several types of surgery. Br J Surg 2013;100:628-37.

13. Adab P, Rouse AM, Mohammed MA, et al. Performance league tables: the NHS deserves better. BMJ 2002;324:95-8.

14. Goldman DA, Brender JD. Are standardized mortality ratios valid for public health data analysis? Stat Med 2000;19:1081-8.

15. van Dishoeck AM, Lingsma HF, Mackenbach JP, et al. Random variation and rankability of hospitals using outcome indicators. BMJ Qual Saf 2011;20:869-74.

16. Soohoo NF, Farng E, Lieberman JR, et al. Factors that predict shortterm complication rates after total hip arthroplasty. Clin Orthop Relat Res 2010;468:2363-71.

17. So-Osman C. Red blood cell transfusion triggers and transfusion alternatives in elective orthopedic hip and knee replacement surgery - a review. International Journal of Clinical Transfusion Medicine 2015;3:47-54.

18. CBO. Guideline Bloodtransfusionpolicy. 2011 https://nvic.nl/cborichtlijn-bloedtransfusie-2011.

19. Voorn VM, Marang-van de Mheen PJ, Wentink MM, et al. Frequent use of blood-saving measures in elective orthopaedic surgery: a 2012 Dutch blood management survey. BMC Musculoskelet Disord 2013;14:230.

20. Dutch Healthcare Inspectorate. Transparant Care (in Dutch). The Hague: IGZ, 2012.

21. Soohoo NF, Tang EY, Krenek L, et al. Variations in the quality of care delivered to patients undergoing total knee replacement at 3 affiliated hospitals. Orthopedics 2011;34:356.

22. Goodnough LT, Maniatis A, Earnshaw P, et al. Detection, evaluation, and management of preoperative anaemia in the elective orthopaedic surgical patient: NATA guidelines. $\mathrm{Br} J$ Anaesth 2011;106:13-22.

23. Jans $\varnothing$, Kehlet $\mathrm{H}$, Hussain $\mathrm{Z}$, et al. Transfusion practice in hip arthroplasty--a nationwide study. Vox Sang 2011;100:374-80.

24. Yoshihara $\mathrm{H}$, Yoneoka D. National trends in the utilization of blood transfusions in total hip and knee arthroplasty. J Arthroplasty 2014;29:1932-7.

25. De Bethune X, Ackaert K, Gillet P, et al. Total hip arthroplasty in Belgium: the contribution of a social health insurer to the debate. Acta Orthop Belg 2014;80:348-56.

26. de Vos M, Graafmans W, Kooistra M, et al. Using quality indicators to improve hospital care: a review of the literature. Int J Qual Health Care 2009;21:119-29.

27. Van Leeuwen A. The best hospitals of 2011 (in Dutch). The Netherlands: Elsevier, 2015.

28. Khanna MP, Hébert PC, Fergusson DA. Review of the clinical practice literature on patient characteristics associated with perioperative allogeneic red blood cell transfusion. Transfus Med Rev 2003;17:110-9.

29. Winemaker M, Petruccelli D, Kabali C, et al. Not all total joint replacement patients are created equal: preoperative factors and length of stay in hospital. Can J Surg 2015;58:160-6.

30. Alsaleh K, Alotaibi GS, Almodaimegh HS, et al. The use of preoperative erythropoiesis-stimulating agents (ESAs) in patients who underwentknee or hip arthroplasty: a meta-analysis of randomized clinical trials. J Arthroplasty 2013;29:1463-72.

31. Alshryda S, Sarda P, Sukeik M, et al. Tranexamic acid in total knee replacement: a systematic review and meta-analysis. J Bone Joint Surg Br 2011;93:1577-85.

32. Bou Monsef J, Boettner F. Blood management may have an impact on length of stay after total hip arthroplasty. Hss $J$ 2014:10:124-30.

33. Chimento GF, Huff T, Ochsner JL, et al. An evaluation of the use of topical tranexamic acid in total knee arthroplasty. J Arthroplasty 2013;28(8 Suppl):74-7.

34. Kotzé A, Carter LA, Scally AJ. Effect of a patient blood management programme on preoperative anaemia, transfusion rate, and outcome after primary hip or knee arthroplasty: a quality improvement cycle. Br J Anaesth 2012;108:943-52

35. van Bodegom-Vos L, Voorn VM, So-Osman C, et al. Cell salvage in hip and knee arthroplasty: A meta-analysis of randomized controlled trials. J Bone Joint Surg Am 2015;97:1012-21.

36. Husted H, Jensen CM, Solgaard S, et al. Reduced length of stay following hip and knee arthroplasty in Denmark 2000-2009: from research to implementation. Arch Orthop Trauma Surg 2012;132:101-4.

37. White JJ, Houghton-Clemmey R, Marval P. Enhanced recovery after surgery (ERAS): an orthopaedic perspective. J Perioper Pract 2013;23:228-32

38. Lirk P, Hollmann MW. Outcome after regional anesthesia: weighing risks and benefits. Minerva Anestesiol 2014;80:610-8. 
39. Marques EM, Jones HE, Elvers KT, et al. Local anaesthetic infiltration for peri-operative pain control in total hip and knee replacement: systematic review and meta-analyses of short- and long-term effectiveness. BMC Musculoskelet Disord 2014;15:220.

40. Opperer M, Danninger T, Stundner O, et al. Perioperative outcomes and type of anesthesia in hip surgical patients: An evidence based review. World J Orthop 2014;5:336-43.

41. Kynaston-Pearson F, Ashmore AM, Malak TT, et al. Primary hip replacement prostheses and their evidence base: systematic review of literature. BMJ 2013;347:f6956.

42. Petis S, Howard JL, Lanting BL, et al. Surgical approach in primary total hip arthroplasty: anatomy, technique and clinical outcomes. Can J Surg 2015;58:128-39.

43. Yi S, Tan J, Chen C, et al. The use of pneumatic tourniquet in total knee arthroplasty: a meta-analysis. Arch Orthop Trauma Surg 2014;134:1469-76.

44. Zan PF, Yang Y, Fu D, et al. Releasing of tourniquet before wound closure or not in total knee arthroplasty: a meta-analysis of randomized controlled trials. J Arthroplasty 2015;30:31-7.

45. Voorn VM, Marang-van de Mheen PJ, So-Osman C, et al. Deimplementation of expensive blood saving measures in hip and knee arthroplasties: study protocol for the LISBOA-II cluster randomized trial. Implement Sci 2014;9:48.

46. Rothman KJ, Boice JD. Epidemiologic analysis with a programmable calculator. Bethesda, MD: National Institutes of Health, 1979:31-2.
47. Houwelingen $\mathrm{H}$, Brand R, Louis T. Empirical bayes methods for monitoring health care quality Bulletin of the ISI2015. 2004. http:// stat.fi/isi99/proceedings/arkisto/varasto/vanh0228.pdf

48. Lingsma HF, Eijkemans MJ, Steyerberg EW. Incorporating natural variation into IVF clinic league tables: The expected rank. BMC Med Res Methodol 2009;9:53.

49. Higgins JP, Thompson SG, Deeks JJ, et al. Measuring inconsistency in meta-analyses. BMJ 2003;327:557-60.

50. Kehlet $\mathrm{H}$. Fast-track hip and knee arthroplasty. The Lancet 2013;381:1600-2.

51. Cunic D, Lacombe S, Mohajer K, et al. Can the Blaylock risk Assessment Screening score (BRASS) predict length of hospital stay and need for comprehensive discharge planning for patients following hip and knee replacement surgery? predicting arthroplasty planning and stay using the BRASS. Can J Surg 2014;57:391-7.

52. Pugely AJ, Gao Y, Martin CT, et al. The effect of resident participation on short-term outcomes after orthopaedic surgery. Clin Orthop Relat Res 2014;472:2290-300.

53. Roberts TT, Vanushkina M, Khasnavis S, et al. Dedicated orthopaedic operating rooms: beneficial to patients and providers alike. J Orthop Trauma 2015;29:e18-23.

54. Tan C, Loo G, Pua YH, et al. Predicting discharge outcomes after total knee replacement using the risk assessment and predictor tool. Physiotherapy 2014;100:176-81.

55. Pitter FT, Jørgensen CC, Lindberg-Larsen M, et al. Postoperative morbidity and discharge destinations after fast-track hip and knee arthroplasty in patients older than 85 years. Anesth Analg 2016;122:1807-15 